\title{
On the probability that two random integers are coprime
}

\author{
Jing Lei* and Joseph B. Kadane \\ Carnegie Mellon University
}

\begin{abstract}
We show that there is a non-empty class of finitely additive probabilities on $\mathbb{N}^{2}$ such that for each member of the class, each set with limiting relative frequency $p$ has probability $p$. Hence, in that context the probability that two random integers are coprime is $6 / \pi^{2}$. We also show that two other interpretations of "random integer," namely residue classes and shift invariance, support any number in $\left[0,6 / \pi^{2}\right]$ for that probability. Finally, we specify a countably additive probability space that also supports $6 / \pi^{2}$.
\end{abstract}

Key words and phrases: number theory, coprime, uniform distribution, finitely additive probability.

\section{INTRODUCTION}

For two integers $a, b$, let $\operatorname{gcd}(a, b)$ be the largest positive integer that evenly divides both $a$ and $b$. It is a well-established result in number theory that

$$
\lim _{n \rightarrow \infty} \frac{\#\left\{(a, b) \in[n]^{2}, \operatorname{gcd}(a, b)=1\right\}}{n^{2}}=\frac{6}{\pi^{2}} .
$$

(Hardy and Wright, 2008, Theorem 331), where $[n]=\{1,2, \ldots, n\}$. They then write "it is natural" to interpret (1.1) as a probability, and conclude (Theorem 332) that

(1.2) the probability that two randomly chosen integers are co-prime is $6 / \pi^{2}$.

The purpose of this paper is to reconcile (1.2) with modern probability theory. To do so, we first introduce the two main frameworks used in probability, and then apply them to (1.2).

\subsection{A brief introduction to probability axioms}

There are two different axiom systems for probability. The first, and most familiar, is countably additive. It starts with a triple of objects: $(\Omega, \mathcal{B}, P)$, where $\Omega$ is the basic set of objects, $\mathcal{B}$ is a $\sigma$-field of subsets of $\Omega$, and $P\{\cdot\}$ is a probability over elements of $\mathcal{B}$ satisfying

(a) $P\{B\} \geq 0$ for all $B \in \mathcal{B}$

$\overline{132}$ Baker Hall, Carnegie Mellon University, Pittsburgh, PA, 15213. (e-mail: jinglei@andrew.cmu.edu; kadane@stat.cmu.edu).

*Research is partially supported by NSF grant DMS- 1553884 . 
(b) $P\{\Omega\}=1$

(c) If $B_{1}, B_{2}, \ldots$ is a countable sequence of disjoint elements of $\mathcal{B}$, then

$$
P\left\{\bigcup_{i=1}^{\infty} B_{i}\right\}=\sum_{i=1}^{\infty} P\left\{B_{i}\right\} .
$$

This is the axiom system advocated by Kolmogorov $(1933)^{1}$. It has the advantage that conditional probabilities obey the tower property:

$$
E(X)=E[E(X \mid Y)],
$$

but the disadvantage that it is defined only on $\mathcal{B}$, and cannot, in general, be extended to the power set of $\Omega$ (Billingsley (1995, Section 3) shows the existence of non-measurable sets).

The second axiom system is finitely additive, replacing (c) above with the condition:

(c') If $B_{1}, B_{2}, \ldots, B_{n}$ is a finite collection of disjoint subsets of $\Omega$, then

$$
P\left\{\bigcup_{i=1}^{n} B_{i}\right\}=\sum_{i=1}^{n} P\left\{B_{i}\right\} .
$$

This has the advantage that a finitely additive distribution can be extended to the power set, but the disadvantage that the tower property does not, in general, hold for them. This sense of probability was particularly advocated by De Finetti $(1937)^{2}$.

Every countably additive probability is finitely additive, but not conversely.

\subsection{Application to coprime integers}

Let $G=\left\{(a, b) \in \mathbb{N}^{2}: \operatorname{gcd}(a, b)=1\right\}$ be the set of pairs of integers that are coprime. We explore the probability of $G$ under various different settings, beginning with limiting relative frequency. Limiting relative frequency cannot be countably additive, since the limiting relative frequency of each pair of integers is zero, but the countable union of them, $\mathbb{N}^{2}$, has limiting relative frequency one. Hence, we consider first finitely additive probabilities on $\mathbb{N}^{2}$, including limiting relative frequency, in Section 2. Sections 3, 4 and 5 apply finitely additive probability on $\mathbb{N}^{2}$ respectively to limiting relative frequency, residue classes and shift invariance. Section 6 proposes a countably additive probability specification that also supports $P\{G\}=6 / \pi^{2}$.

\section{FINITELY ADDITIVE PROBABILITY}

\subsection{General background}

Allowing probabilities that are finitely but not countably additive permits extension to the power set at the cost that many other useful results that are true for countable additive probabilities do not hold. The following theorem, from Kadane and O'Hagan (1995) (relying on results of Bhaskara Rao and Bhaskara Rao (1983)) gives a necessary and sufficient condition for such an extension of a finitely additive probability.

\footnotetext{
${ }^{1}$ For an English translation, see Kolmogorov and Bharucha-Reid (2018).

${ }^{2}$ For an English translation, see Kyburg and Smokler (1980).
} 
TheOREm 2.1. Let $\mathcal{C}$ be any collection of subsets of a set $\Omega$ such that $\Omega \in \mathcal{C}$. Let $\mu$ be a nonnegative real function defined on $\mathcal{C}$ such that $\mu(\Omega)=1$. Then $\mu$ can be extended to a finitely additive probability on all subsets of $\Omega$ if and only if, for all collections of sets $A_{1}, \ldots, A_{a}$ and $B_{1}, \ldots, B_{b}$ in $\mathcal{C}$,

$$
\sum_{i=1}^{a} I_{A_{i}} \leq \sum_{j=1}^{b} I_{B_{j}}
$$

implies that

$$
\sum_{i=1}^{a} \mu\left(A_{i}\right) \leq \sum_{j=1}^{b} \mu\left(B_{j}\right),
$$

where $I_{A}$ is the indicator function of $A$.

A second result, also in Kadane and O'Hagan (1995), gives upper and lower bounds on the probability of a set $D$ (not in general in $\mathcal{C})$ :

TheOREM 2.2. Let $\mathcal{C}$ be any collection of subsets of a set $\Omega$ such that $\Omega \in \mathcal{C}$. Let $\mu$ be a nonnegative real function defined on $\mathcal{C}$ such that $\mu(\Omega)=1$, and let $\mu$ be extendable to a finitely additive probability on all subsets of $\Omega$. Let $\mathcal{M}$ be the set of such extensions. Consider a further set $D \subset \Omega$. Then

$$
\{\mu(D): \mu \in \mathcal{M}\}=[\ell(D, \mathcal{M}), u(D, \mathcal{M})],
$$

where $\ell(D, \mathcal{M})(u(D, \mathcal{M}))$ is the supremum (infimum) of

$$
h^{-1}\left\{\sum_{i=1}^{a} \mu\left(A_{i}\right)-\sum_{j=1}^{b} \mu\left(B_{j}\right)\right\}
$$

over all $A_{1}, A_{2}, \ldots, A_{a}, B_{1}, B_{2}, \ldots, B_{b} \in \mathcal{C}$ and all $a, b, h=1,2,3, \ldots$, such that

$$
\sum_{i=1}^{a} I_{A_{i}}-\sum_{i=1}^{b} I_{B_{j}} \leq(\geq) h I_{D} .
$$

\subsection{Finitely additive uniform probabilities on $\mathbb{N}$}

While there is only one sense of uniformity on a finite set (each element has the same probability), the same is not true on $\mathbb{N}$. Several such senses have been studied in the literature.

1. Limiting relative frequency. Define

$$
\mathcal{C}_{F}=\left\{C \subseteq \mathbb{N}: \quad \lim _{n \rightarrow \infty} \#(C \cap[n]) / n \text { exists }\right\}
$$

be the collection of subsets of $\mathbb{N}$ with a limiting relative frequency. Then it is natural to require $\mu(C)=\lim _{n \rightarrow \infty} \#(C \cap[n]) / n$ for $C \in \mathcal{C}_{F}$. Kadane and O'Hagan (1995) proved that such a $\mu$ is extendable. We denote the collection of all such finitely additive measures by $\mathcal{M}_{F}$.

imsart-sts ver. 2014/10/16 file: coprime_sts.tex date: September 19, 2019 
2. Shift invariance. Another way of defining uniform measure on $\mathbb{N}$ is to require $\mu$ to be shift invariant. Formally, let $s: \mathbb{N} \mapsto \mathbb{N}$ be $s(x)=x+1$. Shift invariance requires $\mu(A)=\mu\left(s^{-1}(A)\right)$ for all $A \subseteq \mathbb{N}$. Denote the set of finitely additive shift invariant probabilities by $\mathcal{M}_{S}$.

3. Residue class. Let $\mathcal{C}_{R}$ be the residue class, consisting of sets of the form

$$
C=R_{j, k}=\{x: x \equiv j \bmod k\}
$$

for some $j \in[k]-1$ and $k \in \mathbb{N}$. Uniformity naturally requires that $\mu\left(R_{j, k}\right)=$ $k^{-1}$ for all $k \in \mathbb{N}$ and $j \in[k]-1$. Kadane and O'Hagan (1995) proved that such a $\mu$ is extendable. We denote the collection of all such extended finitely additive measures by $\mathcal{M}_{R}$.

The results in Kadane and O'Hagan (1995) and Schirokauer and Kadane (2007) jointly imply that that

$$
\mathcal{M}_{F} \subset \mathcal{M}_{S} \subset \mathcal{M}_{R}
$$

and that each of these inclusions is strict.

\subsection{Finitely additive uniform probabilities on $\mathbb{N}^{2}$}

Now we extend the three types of finitely additive uniform probabilities to $\mathbb{N}^{2}$, and present our main result for finitely additive uniform distributions. The proof of the main result and some intermediate claims, such as extendability, are deferred to later sections.

1. Limiting relative frequency on $\mathbb{N}^{2}$. Define

$$
\mathcal{C}_{F}^{2}=\left\{C \subseteq \mathbb{N}^{2}: \lim _{n_{1} \wedge n_{2} \rightarrow \infty} \frac{\#\left(C \cap\left(\left[n_{1}\right] \times\left[n_{2}\right]\right)\right)}{n_{1} n_{2}} \text { exists }\right\},
$$

and $\mu(C)$ be the limit in (2.6) for $C \in \mathcal{C}_{F}^{2}$. Theorem 3.1 below ensures that $\left(\mathcal{C}_{F}^{2}, \mu\right)$ can be extended to $2^{\mathbb{N}^{2}}$. Denote the collection of all such extensions by $\mathcal{M}_{F}^{2}$.

2. Shift invariance on $\mathbb{N}^{2}$. For $j=1,2$, define $s_{j}: \mathbb{N}^{2} \mapsto \mathbb{N}^{2}$ as the shift function that increases the $j$ th coordinate by one. Denote $\mathcal{M}_{S}^{2}$ the set of finitely additive shift invariant probabilities on $\mathbb{N}^{2}$ (i.e., those satisfy $\mu(A)=$ $\mu\left(s_{j}^{-1}(A)\right)$ for all $A \subseteq \mathbb{N}^{2}$ and $\left.j=1,2\right)$.

3. Residue class on $\mathbb{N}^{2}$. Let $\mathcal{C}_{R}^{2}=\mathcal{C}_{R} \times \mathcal{C}_{R}$ be the residue class on $\mathbb{N}^{2}$ and define $\mathcal{M}_{R}^{2}$ be the set of finitely additive probabilities on $\mathbb{N}^{2}$ extended from $\left(\mathcal{C}_{R}^{2}, \mu\right)$ with $\mu\left(R_{j_{1}, k_{1}} \times R_{j_{2}, k_{2}}\right)=\left(k_{1} k_{2}\right)^{-1}$ for all $R_{j_{1}, k_{1}}, R_{j_{2}, k_{2}} \in \mathcal{C}_{R}$.

The following lemma extends its counterpart in $\mathbb{N}$, with an almost identical proof.

Lemma 2.3. $\mathcal{M}_{F}^{2} \subseteq \mathcal{M}_{S}^{2} \subseteq \mathcal{M}_{R}^{2}$.

It is possible to also establish strict inclusions by considering direct products of the examples given in Kadane and O'Hagan (1995); Schirokauer and Kadane (2007). Now we state our main result for finitely additive probabilities.

Theorem 2.4. Let $G=\left\{(x, y) \in \mathbb{N}^{2}: \operatorname{gcd}(x, y)=1\right\}$ be the set of pairs of positive integers that are co-prime. Then

$$
\ell\left(G, \mathcal{M}_{F}^{2}\right)=u\left(G, \mathcal{M}_{F}^{2}\right)=u\left(G, \mathcal{M}_{S}^{2}\right)=u\left(G, \mathcal{M}_{R}^{2}\right)=6 / \pi^{2}
$$


and

$$
\ell\left(G, \mathcal{M}_{S}^{2}\right)=\ell\left(G, \mathcal{M}_{R}^{2}\right)=0,
$$

where the numbers $u(G, \mathcal{M}), \ell(G, \mathcal{M})$ are defined in Theorem 2.2.

Proof of Theorem 2.4. The proof of Theorem 2.4 essentially contains the organization of results proved in the next three sections.

First, Lemma 2.3 implies that

$$
\ell\left(G, \mathcal{M}_{R}^{2}\right) \leq \ell\left(G, \mathcal{M}_{S}^{2}\right) \leq \ell\left(G, \mathcal{M}_{F}^{2}\right) \leq u\left(G, \mathcal{M}_{F}^{2}\right) \leq u\left(G, \mathcal{M}_{S}^{2}\right) \leq u\left(G, \mathcal{M}_{R}^{2}\right) .
$$

To prove (2.7), Theorem 3.2 implies that $\ell\left(G, \mathcal{M}_{F}^{2}\right)=u\left(G, \mathcal{M}_{F}^{2}\right)=6 / \pi^{2}$, while Theorem 4.2 proves that $u\left(G, \mathcal{M}_{R}^{2}\right)=6 / \pi^{2}$. Therefore, (2.7) follows from (2.9).

Next, (2.8) is a direct consequence of (2.9) and Theorem 5.4, which proves $\ell\left(G, \mathcal{M}_{S}^{2}\right)=0$.

Theorem 2.4 implies that if we interpret uniformity by limiting relative frequency, then $G$ has measure $6 / \pi^{2}$ in all finitely additive uniform probabilities on $\mathbb{N}^{2}$. However, if we interpret uniformity by either shift invariance or proportion of residue classes, then the measure of $G$ can be anywhere between 0 and $6 / \pi^{2}$. Both the lower and upper bounds in these cases are new.

\section{LIMITING RELATIVE FREQUENCY}

In this section we prove the subset of claims in Theorem 2.4 involving $\mathcal{M}_{F}^{2}$, as well as extendability of $\left(\mathcal{C}_{F}^{2}, \mu\right)$ where $\mu$ maps $C \in \mathcal{C}_{F}^{2}$ to the limiting relative frequency of $C$ as defined in (2.6).

We first establish extendability.

Theorem 3.1. $\left(\mathcal{C}_{F}^{2}, \mu\right)$ can be extended to $2^{\mathbb{N}^{2}}$.

Proof of Theorem 3.1. Let $A_{1}, \ldots, A_{a}$ and $B_{1}, \ldots, B_{b}$ be elements of $\mathcal{C}_{F}^{2}$ such that

$$
\sum_{i=1}^{a} I_{A_{i}} \leq \sum_{j=1}^{b} I_{B_{j}}
$$

Then for all $k_{1}, k_{2} \in \mathbb{N}$

$$
\sum_{i=1}^{a} \#\left(A_{i} \cap\left(\left[k_{1}\right] \times\left[k_{2}\right]\right)\right) \leq \sum_{j=1}^{b} \#\left(B_{j} \cap\left(\left[k_{1}\right] \times\left[k_{2}\right]\right)\right) .
$$

So

$$
\sum_{i=1}^{a} \lim _{k_{1}<k_{2}, k_{1} \rightarrow \infty} \frac{\#\left(A_{i} \cap\left(\left[k_{1}\right] \times\left[k_{2}\right]\right)\right)}{k_{1} k_{2}} \leq \sum_{j=1}^{b} \lim _{k_{1}<k_{2}, k_{1} \rightarrow \infty} \frac{\#\left(B_{j} \cap\left(\left[k_{1}\right] \times\left[k_{2}\right]\right)\right)}{k_{1} k_{2}},
$$

i.e., $\sum_{i=1}^{a} \mu\left(A_{i}\right) \leq \sum_{j=1}^{b} \mu\left(B_{j}\right)$.

The next result finishes the proof of the subset of claims in Theorem 2.4 involving $\mathcal{M}_{F}^{2}$.

imsart-sts ver. 2014/10/16 file: coprime_sts.tex date: September 19, 2019 
THEOREM 3.2.

$$
\lim _{n_{1} \wedge n_{2} \rightarrow \infty} \frac{\#\left(G \cap\left(\left[n_{1}\right] \times\left[n_{2}\right]\right)\right)}{n_{1} n_{2}}=6 / \pi^{2} .
$$

As a consequence $G \in \mathcal{C}_{F}^{2}$ and $\mu(G)=6 / \pi^{2}$ for all $\mu \in \mathcal{M}_{F}^{2}$.

Theorem 3.2 is a slight generalization of a Theorem in Hardy and Wright (2008), which focuses on the case of $n_{1}=n_{2}$. The proof is similar.

Proof of Theorem 3.2. Without loss of generality, assume $n_{1} \leq n_{2}$. Let $q_{n_{1}, n_{2}}$ be the number of pairs of integers $(a, b) \in\left[n_{1}\right] \times\left[n_{2}\right]$ such that $\operatorname{gcd}(a, b)=1$. Then

$$
\begin{aligned}
q_{n_{1}, n_{2}} & =n_{1} n_{2}-\sum_{p}\left\lfloor n_{1} / p\right\rfloor\left\lfloor n_{2} / p\right\rfloor+\sum_{p_{1} \leq p_{2}}\left\lfloor n_{1} /\left(p_{1} p_{2}\right)\right\rfloor\left\lfloor n_{2} /\left(p_{1} p_{2}\right)\right\rfloor-\ldots \\
& =\sum_{k=1}^{n_{1}} \nu(k)\left\lfloor n_{1} / k\right\rfloor\left\lfloor n_{2} / k\right\rfloor
\end{aligned}
$$

where $\nu(\cdot)$ is the mobius function such that $\nu(k)=(-1)^{s}$ when $k$ is the product of $s$ distinct primes, and $\nu(k)=0$ otherwise $(\nu(1)=1)$.

Because

$$
\begin{aligned}
0 & \leq n_{1} n_{2} / k^{2}-\left\lfloor n_{1} / k\right\rfloor\left\lfloor n_{2} / k\right\rfloor \\
& =\left(n_{2} / k-\left\lfloor n_{2} / k\right\rfloor\right)\left(n_{1} / k\right)+\left(n_{1} / k-\left\lfloor n_{1} / k\right\rfloor\right)\left\lfloor n_{2} / k\right\rfloor \leq\left(n_{1}+n_{2}\right) / k,
\end{aligned}
$$

we have

$$
\begin{aligned}
\left|\sum_{k=1}^{n_{1}} \nu(k)\left(n_{1} n_{2} / k^{2}\right)-q_{n_{1}, n_{2}}\right| & =\left|\sum_{k=1}^{n_{1}} \nu(k)\left(n_{1} n_{2} / k^{2}-\left\lfloor n_{1} / k\right\rfloor\left\lfloor n_{2} / k\right\rfloor\right)\right| \\
& \leq\left(n_{1}+n_{2}\right) \sum_{k=1}^{n_{1}}(1 / k)=o\left(n_{1} n_{2}\right) .
\end{aligned}
$$

So

$$
\frac{q_{n_{1}, n_{2}}}{n_{1} n_{2}}=\sum_{k=1}^{n_{1}} \nu(k) k^{-2}+o(1) \rightarrow 6 / \pi^{2} .
$$

The results of this section give a framework that justifies Hardy and Wright's claim that (1) implies (2). In this connection, the proof of (1) offered by Abrams and Paris (1992) is not correct, because it relies on countable additivity of limiting relative frequency.

\section{RESIDUE CLASSES}

In this section we first address the extendability of $\mathcal{C}_{R}^{2}$, and then prove that $u\left(G, \mathcal{M}_{R}\right)=6 / \pi^{2}$. The lower bound $\ell\left(G, \mathcal{M}_{R}^{2}\right)=0$ will be proved as a consequence of $\ell\left(G, \mathcal{M}_{S}^{2}\right)=0$, which is established in the next section.

TheORem 4.1. Let $\mu$ be a function defined on $\mathcal{C}_{R}^{2}$ satisfying $\mu\left(R_{j_{1}, k_{1}} \times R_{j_{2}, k_{2}}\right)=$ $1 / k_{1} k_{2}$ for all $j_{1}, j_{2}, k_{1}, k_{2} \in \mathbb{N}$. Then $\mu$ can be extended to $2^{\mathbb{N}^{2}}$.

imsart-sts ver. 2014/10/16 file: coprime_sts.tex date: September 19, 2019 
Proof of Theorem 4.1. We first establish a 1-1 map between $R_{j_{1}, k_{1}} \times R_{j_{2}, k_{2}}$ and $R_{j_{2} k_{1}+j_{1}, k_{1} k_{2}}$, which is realized by writing an arbitrary $k \in\left[k_{1} k_{2}\right]-1$ uniquely as $k=j_{2} k_{1}+j_{1}$ for $j_{1} \in\left[k_{1}\right]-1$ and $j_{2} \in\left[k_{2}\right]-1$.

Now each element of $\mathcal{C}_{R}^{2}$ can be mapped 1-1 to an element of $\mathcal{C}_{R}$. By the result of Kadane and O'Hagan (1995), the set of residue classes can be extended. Therefore so can $\mathcal{C}_{R}^{2}$.

The rest of this section focuses on proving $u\left(G, \mathcal{M}_{R}\right)=6 / \pi^{2}$. We begin by introducing a general way of identifying $u\left(D, \mathcal{M}_{R}^{2}\right)$ for arbitrary $D \subseteq \mathbb{N}^{2}$.

TheOrem 4.2. For all $D \subseteq \mathbb{N}^{2}$,

$$
u\left(D, \mathcal{M}_{R}^{2}\right)=\inf _{t_{1}, t_{2}} \frac{r_{t_{1}, t_{2}}(D)}{t_{1} t_{2}},
$$

where

$$
r_{t_{1}, t_{2}}(D)=\#\left\{\left(k_{1}, k_{2}\right) \in\left(\left[t_{1}\right]-1\right) \times\left(\left[t_{2}\right]-1\right): D \cap\left(R_{k_{1}, t_{1}} \times R_{k_{2}, t_{2}}\right) \neq \emptyset\right\} .
$$

Proof of Theorem 4.2. According to Theorem 2.2,

$$
u\left(D, \mathcal{M}_{R}^{2}\right)=\inf h^{-1}\left[\sum_{i=1}^{a} \mu\left(A_{i}\right)-\sum_{j=1}^{b} \mu\left(B_{j}\right)\right]
$$

where the inf is taken over all $A_{1}, \ldots, A_{a}, B_{1}, \ldots, B_{b} \in \mathcal{C}_{R}^{2}$ and $h=1,2,3, \ldots$ such that

$$
\sum_{i=1}^{a} I_{A_{i}}-\sum_{j=1}^{b} I_{B_{j}} \geq h I_{D}
$$

Let $t=\left(t_{1}, t_{2}\right)$ be the pair of least common multiples of the moduli pairs of the residue sets $A_{1}, \ldots, A_{a}, B_{1}, \ldots, B_{b}$. Then

$$
h I_{D} \leq \sum_{i=1}^{a} I_{A_{i}}-\sum_{j=1}^{b} I_{B_{j}}=\sum_{k_{1}=0}^{t_{1}-1} \sum_{k_{2}=0}^{t_{2}-1} d_{k_{1}, k_{2}} I_{R_{k_{1}, t_{1}} \times R_{k_{2}, t_{2}}}
$$

for some integers $d_{0,0}, d_{0,1}, \ldots, d_{t_{1}-1, t_{2}-1}$.

Thus

$$
u\left(D, \mathcal{M}_{R}^{2}\right)=\inf h^{-1} \sum_{k_{1}=0}^{t_{1}-1} \sum_{k_{2}=0}^{t_{2}-1} d_{k_{1}, k_{2}}\left(\frac{1}{t_{1} t_{2}}\right)=\inf h^{-1}\left(\frac{1}{t_{1} t_{2}}\right) \sum_{k_{1}=0}^{t_{1}-1} \sum_{k_{2}=0}^{t_{2}-1} d_{k_{1}, k_{2}},
$$

where the inf is taken over all $\left(t_{1}, t_{2}\right)$ and $\left(d_{k_{1}, k_{2}}: k_{1} \in\left[t_{1}\right]-1, k_{2} \in\left[t_{2}\right]-1\right)$ such that (4.2) holds.

For given $\left(t_{1}, t_{2}\right)$, the right hand side of the above equation is minimized by setting $d_{k_{1}, k_{2}}=h$ if $D \cap\left(R_{k_{1}, t_{1}} \times R_{k_{2}, t_{2}}\right) \neq \emptyset$ and $d_{k_{1}, k_{2}}=0$ otherwise.

Lemma 4.3. If $(x, y) \in G$, then for every $n \in \mathbb{N}$ there exists $a \in \mathbb{N}$ such that $\operatorname{gcd}(a x+y, n)=1$.

imsart-sts ver. 2014/10/16 file: coprime_sts.tex date: September 19, 2019 
Proof of Lemma 4.3. Let $p_{1}, \ldots, p_{\ell}, q_{1}, \ldots, q_{k}, r_{1}, \ldots, r_{h}$ be all distinct prime factors of $n$ such that

$$
\begin{aligned}
& x \equiv\left(0, \ldots, 0, a_{1}, \ldots, a_{k}, c_{1}, \ldots, c_{h}\right) \bmod \left(p_{1}, \ldots, p_{\ell}, q_{1}, \ldots, q_{k}, r_{1}, \ldots, r_{h}\right) \\
& y \equiv\left(b_{1}, \ldots, b_{\ell}, 0, \ldots, 0, d_{1}, \ldots, d_{h}\right) \bmod \left(p_{1}, \ldots, p_{\ell}, q_{1}, \ldots, q_{k}, r_{1}, \ldots, r_{h}\right)
\end{aligned}
$$

where $1 \leq a_{j} \leq q_{j}-1,1 \leq c_{j} \leq r_{j}-1,1 \leq d_{j} \leq r_{j}-1,1 \leq b_{j} \leq p_{j}-1$, for all $j$.

Then one can pick any $a$ that satisfies

$$
a \equiv(0, \ldots, 0,1, \ldots, 1,0, \ldots, 0) \bmod \left(p_{1}, \ldots, p_{\ell}, q_{1}, \ldots, q_{k}, r_{1}, \ldots, r_{h}\right) \text {. }
$$

Existence of such an $a$ is guaranteed by the Chinese remainder theorem.

Lemma 4.4. Let $k_{1}, k_{2}$ be two positive integers, and $\left(j_{1}, j_{2}\right) \in\left[k_{1}\right] \times\left[k_{2}\right]$. Then $G \cap\left(R_{j_{1}, k_{1}} \times R_{j_{2}, k_{2}}\right) \neq \emptyset$ if and only if $\operatorname{gcd}\left(j_{1}, j_{2}, k_{1}, k_{2}\right)=1$.

Proof of Lemma 4.4. The necessity is obvious. We only need to prove sufficiency.

For $i=1,2$, let $p_{i}=\operatorname{gcd}\left(k_{i}, j_{i}\right), k_{i}=p_{i} r_{i}, j_{i}=p_{i} s_{i}$. By construction and the assumption that $\operatorname{gcd}\left(k_{1}, j_{1}, k_{2}, j_{2}\right)=1$ we have

$$
\operatorname{gcd}\left(p_{1}, p_{2}\right)=\operatorname{gcd}\left(r_{1}, s_{1}\right)=\operatorname{gcd}\left(r_{2}, s_{2}\right)=1 .
$$

Then apply Lemma 4.3 to $(n, x, y)=\left(p_{2}, r_{1}, s_{1}\right)$, there exists $a_{1} \in \mathbb{N}$ such that

$$
\operatorname{gcd}\left(p_{2}, a_{1} r_{1}+s_{1}\right)=1 \text {. }
$$

Apply Lemma 4.3 again to $(n, x, y)=\left(p_{1}\left(a_{1} r_{1}+s_{1}\right), r_{2}, s_{2}\right)$, there exists an $a_{2} \in \mathbb{N}$ such that

$$
\operatorname{gcd}\left[p_{1}\left(a_{1} r_{1}+s_{1}\right), a_{2} r_{2}+s_{2}\right]=1 .
$$

Now combine $(4.3),(4.4)$ and that $\operatorname{gcd}\left(p_{1}, p_{2}\right)=1$ we have

$$
\operatorname{gcd}\left(a_{1} k_{1}+j_{1}, a_{2} k_{2}+j_{2}\right)=1
$$

Theorem 4.5. $u\left(G, \mathcal{M}_{R}^{2}\right)=6 / \pi^{2}$.

Proof of Theorem 4.5. Let $\left(k_{1}, k_{2}\right) \in \mathbb{N}^{2}$ and denote $\operatorname{cd}\left(k_{1}, k_{2}\right)$ the set of prime common divisors of $k_{1}$ and $k_{2}$. Then Lemma 4.4 implies that $G \cap\left(R_{j_{1}, k_{1}} \times\right.$ $\left.R_{j_{2}, k_{2}}\right) \neq \emptyset$ if and only if $j_{1}, j_{2}$ are not both divisible by any $p \in \operatorname{cd}\left(k_{1}, k_{2}\right)$. As a result,

$$
\frac{r_{k_{1}, k_{2}}}{k_{1} k_{2}}=\prod_{p \in \operatorname{cd}\left(k_{1}, k_{2}\right)}\left(1-p_{j}^{-2}\right) .
$$

Now apply Theorem 4.2,

$$
u\left(G, \mathcal{M}_{R}^{2}\right)=\inf _{k_{1}, k_{2}} \prod_{p \in \operatorname{cd}\left(k_{1}, k_{2}\right)}\left(1-p^{-2}\right)=\prod_{p \text { prime }}\left(1-p^{-2}\right)=\frac{6}{\pi^{2}} .
$$

imsart-sts ver. 2014/10/16 file: coprime_sts.tex date: September 19, 2019 


\section{SHIFT INVARIANCE}

Combining Lemma 2.3 with Theorem 3.2 and Theorem 4.5 we have

$$
u\left(G, \mathcal{M}_{S}^{2}\right)=6 / \pi^{2} .
$$

Therefore, the proof of Theorem 2.4 will be complete if we can show

$$
\ell\left(G, \mathcal{M}_{S}^{2}\right)=0
$$

which is the focus of the current section.

We prove the claim in a more general setting. Let $d \geq 2$ be a positive integer. For $1 \leq i \leq d$, let $s_{i}: \mathbb{N}^{d} \mapsto \mathbb{N}^{d}$ be the shift operator in the $i$ th coordinate:

$$
s_{i}\left(a_{1}, \ldots, a_{d}\right)=\left(a_{1}, \ldots, a_{i}+1, \ldots, a_{d}\right) .
$$

We call a function $\mu: 2^{\mathbb{N}^{d}} \mapsto \mathbb{R}$ shift-invariant if $\mu(A)=\mu\left(s_{i}^{-1}(A)\right)$ for all $A \subseteq \mathbb{N}^{d}$ and all $1 \leq i \leq d$.

Following ideas in Schirokauer and Kadane (2007), we study shift-invariant functions by constructing linear functionals on $\ell^{\infty}\left(\mathbb{N}^{d}\right)$ with certain desirable properties. Recall the definition of $\ell^{\infty}\left(\mathbb{N}^{d}\right)$.

$$
\ell^{\infty}\left(\mathbb{N}^{d}\right)=\left\{x=\left(x(a) \in \mathbb{R}: a \in \mathbb{N}^{d}\right): \sup _{a \in \mathbb{N}^{d}}|x(a)|<\infty\right\} .
$$

Then $\ell^{\infty}\left(\mathbb{N}^{d}\right)$ is a Banach space equipped with norm $\|x\|=\sup _{a \in \mathbb{N}^{d}}|x(a)|$.

LEMma 5.1. There exists a linear functional $\Phi$ on $\ell^{\infty}\left(\mathbb{N}^{d}\right)$ such that

1. $\Phi$ is shift-invariant: $\Phi(x)=\Phi\left(S_{i} x\right)$ for all $i \in[d]$, where $\left(S_{i} x\right)(a)=x\left(s_{i}(a)\right)$ for all $a \in \mathbb{N}^{d}$;

2. $\Phi$ is positive: $\Phi \geq 0$ whenever $x(a) \geq 0$ for all $a \in \mathbb{N}^{d}$;

3. $\Phi$ is normalized: $\Phi(\mathbf{1})=1$ where $\mathbf{1}$ is the constant-1 vector.

Proof of Lemma 5.1. Consider the linear subspace of $\ell^{\infty}\left(\mathbb{N}^{d}\right)$ given by

$$
W \stackrel{\text { def }}{=}\left\{\sum_{i=1}^{d}\left(S_{i} x_{i}-x_{i}\right): x_{i} \in \ell^{\infty}\left(\mathbb{N}^{d}\right)\right\} .
$$

Then we can claim that $\bar{W}$ and $\mathbb{R} \mathbf{1}$ intersect trivially. To see this, let $c \neq 0$ and $w=\sum_{i=1}^{d}\left(S_{i} x_{i}-x_{i}\right)$, then

$$
\|w+c \mathbf{1}\| \geq \frac{1}{n^{d}} \sum_{a \in[n]^{d}}|w(a)+c| \geq \frac{1}{n^{d}}\left|\sum_{a \in[n]^{d}}(w(a)+c)\right| \rightarrow|c|
$$

as $n \rightarrow \infty$.

Now let $\Phi_{0}$ be a linear functional on $W \oplus \mathbb{R} \mathbf{1}$ given by

$$
\Phi_{0}(w+c \mathbf{1})=c .
$$

By (5.1), $\left\|\Phi_{0}\right\| \leq 1$. By Hahn-Banach Theorem, there exists an extension $\Phi$ of $\Phi_{0}$ to $\ell^{\infty}\left(\mathbb{N}^{d}\right)$ such that $\|\Phi\| \leq 1$.

Now we check that such a linear functional $\Phi$ satisfies the requirements of the claim.

imsart-sts ver. 2014/10/16 file: coprime_sts.tex date: September 19, 2019 
1. Shift-invariance: by linearity $\Phi\left(S_{i} x\right)-\Phi(x)=\Phi\left(S_{i} x-x\right)=0$.

2. Normalized: by construction.

3. Positivity: if $x \in \ell^{\infty}\left(\mathbb{N}^{d}\right)$ is positive, then we can write $x=c y$ for some $c>0$ and $\|y\| \leq 1$, and

$$
\Phi(x)=c \Phi(y)=c(1-\Phi(\mathbf{1}-y)) \geq c(1-\|\mathbf{1}-y\|) \geq 0
$$

The usefulness of Lemma 5.1 is the following general construction of shiftinvariant probability measures on $2^{\mathbb{N}^{d}}$.

For $X, A \subseteq \mathbb{N}^{d}$, define $s^{-A}(X)=\bigcup_{a \in A} s^{-a}(X)$, where $s^{-a}(X)=s_{1}^{-a_{1}}\left(\cdots s_{d}^{-a_{d}}(X)\right)$ for $a=\left(a_{1}, \ldots a_{d}\right) \in \mathbb{N}^{d}$.

Lemma 5.2. Let $\mu_{1}$ be a finitely additive probability on $2^{\mathbb{N}^{d}}$. Define $\mu: 2^{\mathbb{N}^{d}} \mapsto$ $\mathbb{R}$ as

$$
\mu(Z)=\Phi\left\{\left[\mu_{1}\left(s^{-a}(Z)\right): a \in \mathbb{N}^{d}\right]\right\}
$$

Then $\mu$ is a finitely additive, shift-invariant probability on $2^{\mathbb{N}^{d}}$.

Proof of Lemma 5.2. First $\mu_{1}\left(s^{-a}(Z)\right) \in[0,1]$ for all $a \in \mathbb{R}^{d}$, by positivity and normalization of $\Phi$ we have $\mu(Z) \in[0,1]$ for all $Z$.

Second, when $Z=\mathbb{N}^{d}$ we have $s^{-a}(Z)=\mathbb{N}^{d}$ for all $a \in \mathbb{N}^{d}$, and hence $\mu(Z)=$ $\Phi(\mathbf{1})=1$.

Third, if $Z_{1}, Z_{2} \subseteq \mathbb{N}^{d}$ are disjoint, then $s^{-a}\left(Z_{1} \cup Z_{2}\right)=s^{-a}\left(Z_{1}\right) \cup s^{-a}\left(Z_{2}\right)$ and $s^{-a}\left(Z_{1}\right) \cap s^{-a}\left(Z_{2}\right)=\emptyset$. Then finite additivity of $\mu$ follows from linearity of $\Phi$.

Finally, for $i \in[d], s^{-a}\left(s_{i}^{-1}(Z)\right)=s^{-s_{i}(a)}(Z)$, so the shift-invariance of $\mu$ follows from the shift-invariance of $\Phi$ (Property 1 of Lemma 5.1).

Lemma 5.3. For $X \subseteq \mathbb{N}^{d}$, the following are equivalent.

1. $s^{-A}(X) \neq \mathbb{N}^{d}$ for any finite set $A \subset \mathbb{N}^{d}$.

2. There is a shift-invariant finitely-additive probability $\mu$ on $2^{\mathbb{N}^{d}}$ such that $\mu(X)=0$.

Proof of Lemma 5.3. " $2 \Rightarrow 1$ ": If $\mu(X)=0$, then $\mu\left(s^{-a}(X)\right)=0$ for every a. Hence $\mu\left(s^{-A}(X)\right)=0$ for any finite $A$.

" $1 \Rightarrow 2$ ": Let $\mathcal{C}$ be a family consisting of $\mathbb{N}^{d}$ and all sets of the form $s^{-A}(X)$ with finite $A$. Let $\mu_{0}: \mathcal{C} \mapsto \mathbb{R}^{+}$be defined as $\mu_{0}\left(\mathbb{N}^{d}\right)=1, \mu_{0}(Y)=0$ if $Y \neq \mathbb{N}^{d}$. The assumption that $s^{-A}(X) \neq \mathbb{N}^{d}$ for any finite set $A$ implies that, according to Theorem 1 of Kadane and O'Hagan (1995), $\mu_{0}$ can be extended to $2^{\mathbb{N}^{d}}$. Let $\mu_{1}$ be such an extended finitely additive probability and let

$$
\mu(Z)=\Phi\left(\left(\mu_{1}\left(s^{-a}(Z)\right): a \in \mathbb{N}^{d}\right)\right)
$$

where $\Phi$ is the functional constructed in Lemma 5.1 .

Lemma 5.2 ensures that $\mu$ is a shift-invariant finitely additive probability. On the other hand, $\mu_{1}\left(s^{-a}(X)\right)=\mu_{0}\left(s^{-a}(X)\right)=0$ for all $a \in \mathbb{N}^{d}$. By construction, $\mu(X)=\Phi(\mathbf{0})=0$.

THEOREM 5.4. $\ell\left(G, \mathcal{M}_{S}^{2}\right)=0$. 
Proof of Theorem 5.4. According to Lemma 5.3, it suffices to prove that $s^{-A}(G) \neq \mathbb{N}^{2}$ for every finite $A \subset \mathbb{N}^{2}$.

Let $\left(a_{1}, b_{1}\right),\left(a_{2}, b_{2}\right), \ldots,\left(a_{m}, b_{m}\right)$ be enumeration of all elements of $A$. Let $p_{1}, \ldots, p_{m}$ be $m$ arbitrary distinct prime numbers. By Chinese remainder theorem there exist $a, b \in \mathbb{N}$ such that

$$
\begin{aligned}
a+a_{i} & \equiv 0 \bmod p_{i}, \quad \forall i \in[m], \\
b+b_{i} & \equiv 0 \bmod p_{i}, \quad \forall i \in[m] .
\end{aligned}
$$

Then $(a, b) \notin s^{-A}(G)$.

\section{COUNTABLY ADDITIVE PROBABILITY}

In order to keep countable additivity in the probability, we must work with a smaller $\sigma$-field of subsets of $\mathbb{N}^{2}$.

For $i \in \mathbb{N}$ let $p_{i}$ be the $i$ th prime number and define

$$
A_{i}=\left\{x \in \mathbb{N}: x \equiv 0 \bmod p_{i}\right\} .
$$

For finite disjoint subsets $I, J$ of $\mathbb{N}$ let

$$
A_{I, J}=\left(\cap_{i \in I} A_{i}\right) \bigcap\left(\cap_{i \in J} A_{i}^{c}\right)
$$

be the set of positive integers divisible by prime numbers in $I$ but not by those in $J$. It is allowed to have $I=J=\emptyset$, and we define $A_{\emptyset, \emptyset}=\mathbb{N}$. Define

$$
\mathcal{C}=\left\{\bigcup_{k=1}^{K} A_{I_{k}, J_{k}}: K \in \mathbb{N}, I_{k} \cap J_{k}=\emptyset,\left|I_{k}\right|,\left|J_{k}\right|<\infty\right\} \bigcup\{\emptyset\}
$$

Lemma 6.1. $\quad \mathcal{C}$ is a field of subsets of $\mathbb{N}$.

Proof of Lemma 6.1. Consider $\mathcal{A}=\{0,1\}^{\mathbb{N}}$. For finite disjoint $I, J \subset \mathbb{N}$, we can represent $A_{I, J}$ as a subset of $\mathcal{A}$ by $A_{I, J} \Leftrightarrow\{0\}^{I} \times\{1\}^{J} \times\{0,1\}^{(I \cup J)^{c}}$. For example when $I=\{2\}, J=\{1,3\}$, then the corresponding subset of $\mathcal{A}$ is $\left\{x \in\{0,1\}^{\mathbb{N}}: x_{1}=0, x_{2}=1, x_{3}=0\right\}$, the cylinder in $\{0,1\}^{\mathbb{N}}$ with base $(0,1,0)$.

It is easy to check $\emptyset$ and $A_{\emptyset, \emptyset}=\mathbb{N}$ are in $\mathcal{C}$. We proceed to make the following three observations.

(a) $\mathcal{C}$ is closed under finite unions.

Let

$$
C_{1}=\bigcup_{k=1}^{K_{1}} A_{I_{k}^{1}, J_{k}^{1}} \text { and } C_{2}=\bigcup_{k=K_{1}+1}^{K_{2}} A_{I_{k}^{2}, J_{k}^{2}}
$$

and

$$
\begin{array}{lll}
I_{k}=I_{k}^{1} \text { and } J_{k}=J_{k}^{1} & \text { for } & 1 \leq k \leq K_{1} \\
I_{k}=I_{k}^{2} \text { and } J_{k}=J_{k}^{2} & \text { for } & K_{1}+1 \leq k \leq K_{2} .
\end{array}
$$

Then

$$
C_{1} \cup C_{2}=\cup_{k=1}^{K_{1}+K_{2}} A_{I_{k}, J_{k}} \in \mathcal{C}
$$


(b) $A_{I, J}^{c} \in \mathcal{C}$.

Now assume $(I, J) \neq(\emptyset, \emptyset)$. Use the product representation to write $A_{I, J}^{c}$ :

$$
A_{I, J}^{c}=\left[\{0,1\}^{I \cup J} \backslash\left(\{0\}^{I} \times\{1\}^{J}\right)\right] \times\{0,1\}^{(I \cup J)^{c}} .
$$

and

$$
\{0,1\}^{I \cup J} \backslash\{0\}^{I} \times\{1\}^{J}=\bigcup_{I^{\prime} \subseteq(I \cup J), I^{\prime} \neq I}\{0\}^{I^{\prime}} \times\{1\}^{(I \cup J) \backslash I^{\prime}} .
$$

This shows that $A_{I, J}^{c}=\bigcup_{I^{\prime} \subseteq(I \cup J), I^{\prime} \neq I} A_{I^{\prime},(I \cup J) \backslash I^{\prime}} \in \mathcal{C}$.

(c) $A_{I_{1}, J_{1}} \cap A_{I_{2}, J_{2}} \in \mathcal{C}$.

For finite disjoint $\left(I_{j}, J_{j}\right),(j=1,2)$, let $T=\cup\left(I_{1}, J_{1}, I_{2}, J_{2}\right)$. We consider the augmented representation of $A_{I_{1}, J_{1}}$ and $A_{I_{2}, J_{2}}$

$$
\begin{aligned}
& A_{I_{1}, J_{1}}=\{0\}^{I_{1}} \times\{1\}^{J_{1}} \times\{0,1\}^{T \backslash\left(I_{1} \cup J_{1}\right)} \times\{0,1\}^{T^{c}} \\
& A_{I_{2}, J_{2}}=\{0\}^{I_{2}} \times\{1\}^{J_{2}} \times\{0,1\}^{T \backslash\left(I_{2} \cup J_{2}\right)} \times\{0,1\}^{T^{c}}
\end{aligned}
$$

Let $B_{j}=\{0\}^{I_{j}} \times\{1\}^{J_{j}} \times\{0,1\}^{T \backslash\left(I_{j} \cup J_{j}\right)}$ for $j=1,2$. Then each $B_{j}$ is a subset of $\{0,1\}^{T}$, which is a finite set. Now let $C=B_{1} \cap B_{2}$, then $C$ is a subset of $\{0,1\}^{T}$. So there exists a subset $\mathcal{I} \subseteq T$, such that

$$
C=\bigcup_{I^{\prime} \in \mathcal{I}}\{0\}^{I^{\prime}} \times\{1\}^{T \backslash I^{\prime}}
$$

Since $T$ is finite, the union in the above expression for $C$ is finite. Thus we proved that $A_{I_{1}, J_{1}} \cap A_{I_{2}, J_{2}} \in \mathcal{C}$.

The three observations (a-c) are sufficient to imply further claims such as that $\mathcal{C}$ is closed under complement, which concludes the proof.

Remark. Note that although $\mathcal{C}$ has an isomorphism between the subsets of $\mathbb{N}$ and those in $\mathcal{A}$, the generated $\sigma$-fields are different. In fact $\cap_{i=1}^{\infty} A_{\{i\}, \emptyset}=\emptyset$, but $\cap_{i=1}^{\infty}\{0\}^{\{i\}} \times\{0,1\}^{\mathbb{N} \backslash\{i\}}=\{0\}^{\mathbb{N}} \neq \emptyset$.

Now we are ready to define the uniform probability measure on $\mathcal{C}$. Let $P: \mathcal{C} \mapsto$ $[0,1]$ be that if $C=\cup_{k=1}^{K} A_{I_{k}, J_{k}}$ for disjoint sets $\left\{A_{I_{k}, J_{k}}: 1 \leq k \leq K\right\}$, then

$$
P\{C\}=\sum_{k=1}^{K} P\left\{A_{I_{k}, J_{k}}\right\}
$$

with

$$
P\left\{A_{I, J}\right\}=\prod_{i \in I} p_{i}^{-1} \prod_{i \in J}\left(1-p_{i}^{-1}\right) .
$$

We further define $P\{\emptyset\}=0$ and $P\{\mathbb{N}\}=1$.

Equation (6.1) reflects the uniformity of $P$ : For distinct prime numbers $p$ and $q$

(i) the probability of being divisible by a prime number $p$ is $p^{-1}$;

(ii) being divisible by $p$ and being divisible by $q$ are independent events.

Theorem 6.2. $P$ is a probability on $\mathcal{C}$ and can be uniquely extended to $\mathcal{F}=$ $\sigma(\mathcal{C})$.

imsart-sts ver. 2014/10/16 file: coprime_sts.tex date: September 19, 2019 
Proof of Theorem 6.2. We only need to prove countable additivity of $P$ on $\mathcal{C}$. The second part follows from Carathéodory's extension.

Let $A_{I, J}=\bigcup_{k=1}^{\infty} A_{I_{k}, J_{k}}$, where $\left\{A_{I_{k}, J_{k}}: k \geq 1\right\}$ are disjoint with $I_{k}, J_{k}$ finite and disjoint. Now define $Q$ to be the product measure on $\mathcal{A}$ with marginal $Q_{i}$ being Bernoulli $\left(1-p_{i}^{-1}\right)$. The existence and uniqueness of $Q$ is guaranteed by Kolmogorov's extension.

Then $P$ and $Q$ agree on $\mathcal{C}$. Since $Q$ is a probability measure we have

$$
P\left\{A_{I, J}\right\}=Q\left\{A_{I, J}\right\}=\sum_{k=1}^{\infty} Q\left\{A_{I_{k}, J_{k}}\right\}=\sum_{k=1}^{\infty} P\left\{A_{I_{k}, J_{k}}\right\}
$$

Theorem 6.3. Let $P_{2}$ be the product measure of $P$ on $\mathbb{N}^{2}$. Then

$$
P_{2}\{G\}=6 / \pi^{2} \text {. }
$$

Proof of Theorem 6.3. $\operatorname{gcd}(x, y)=1$ if and only if $(x, y) \in\left(A_{\{i\}, \emptyset} \times A_{\{i\}, \emptyset}\right)^{c}$ for all $i$. By independence between $A_{\{i\}, \emptyset}$ as $i$ changes,

$$
P_{2}\{G\}=\prod_{i=1}^{\infty}\left(1-p_{i}^{-2}\right)=\frac{6}{\pi^{2}} . \square
$$

\section{CONCLUSION}

The probability assigned to the set of $G$ of relatively prime integers depends on the sense of "uniform" probability being used. When the class of finitely additive probabilities defined by relative frequency is used, $P(G)=6 / \pi^{2}$. Similarly, when the countably additive probability defined in Section $6, P\{G\}=6 / \pi^{2}$ is the only value supported. However, when the finitely additive classes defined by shift invariance or residue classes are involved, there are elements of those classes satisfying $P\{G\}=x$ if and only if $x \in\left[0,6 / \pi^{2}\right]$.

\section{ACKNOWLEDGEMENTS}

The authors would like to thank Boris Bukh for pointing out Theorem 5.4 and the idea of its proof.

\section{REFERENCES}

Abrams, A. and PARIs, M. (1992). The probability that $(\mathrm{a}, \mathrm{b})=1$. The College Math Journal 2347.

Bhaskara RaO, K. P. S. and Bhaskara RaO, M. (1983). Theory of Charges. Academic Press, New York.

Billingsley, P. (1995). Probability and Measure. John Wiley\&Sons, New York.

De Finetti, B. (1937). La prévision: ses lois logiques, ses sources subjectives. In Annales de l'institut Henri Poincaré 7 1-68.

Hardy, G. H. and Wright, E. M. (2008). An Introduction to the Theory of Numbers, 6th ed. Oxford University Press.

Kadane, J. B. and O'Hagan, A. (1995). Using Finitely Additive Probability: Uniform Distributions on the Natural Numbers. Journal of the American Statistical Association 90 626-631.

Kolmogorov, A. N. (1933). Grundbegriffe der Wahrscheinlichkeitsrechnung. Julius Springer, Berlin.

Kolmogorov, A. N. and Bharucha-Reid, A. T. (2018). Foundations of the theory of probability: Second English Edition. Courier Dover Publications.

Kyburg, H. E. and Smokler, H. E. (1980). Studies in subjective probability.

Schirokauer, O. and KadAne, J. B. (2007). Uniform Distributions on the Natural Numbers. Journal of Theoretical Probability 20 429-441.

imsart-sts ver. 2014/10/16 file: coprime_sts.tex date: September 19, 2019 